\title{
Using endoscopic ultrasound-guided fine needle aspiration to diagnose mediastinal cryptococcosis
}

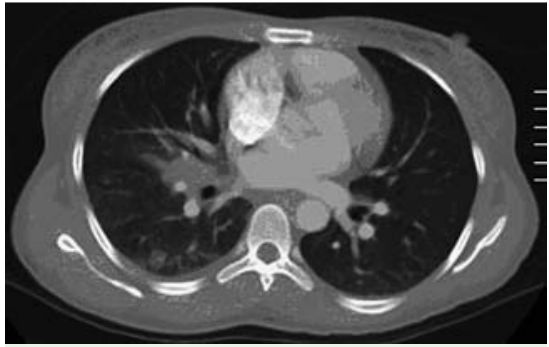

Fig. 1 Computed tomography (CT) scan of the chest, showing hilar adenopathy.

A 33-year-old woman from Zimbabwe with AIDS and a CD4 count of 6 cells $/ \mathrm{mm}^{3}$, non-compliant with antiretroviral therapy, presented with severe headaches, vomiting, fevers, and a productive cough. Physical examination revealed tachycardia, a temperature of $39.4^{\circ} \mathrm{C}$, clear lungs, and an absence of meningeal signs. Laboratory studies showed a white blood cell count of 4.8 and hemoglobin of $10.1 \mathrm{~g} / \mathrm{dL}$. A lumbar puncture was consistent with cryptococcal meningitis (cryptococcal antigen titer $1: 32768$ ), and the patient had positive blood cultures growing Cryptococcus neoformans. The admitting chest radiograph was unremarkable.

Amphotericin B and flucytosine were given. Her repeat lumbar puncture 2 weeks later showed an unchanged cryptococcal antigen titer, and she continued to have high fevers $\left(39.4-40^{\circ} \mathrm{C}\right)$ and cough. Tuberculosis was ruled out. A computed tomography scan of the chest, abdomen, and pelvis revealed bilateral multiple moderately enlarged mediastinal and hilar lymphadenopathy, patchy infiltrate in the right lung, and mild hepatomegaly (๑ Fig. 1).

Endoscopic ultrasound (EUS) was performed to evaluate the mediastinal lymphadenopathy. Images showed a large heterogeneous hypoechoic mass extending from the subcarinal space to the distal peri-esophageal space ( $\boldsymbol{F i g . 2}$ ). The mass was sampled by fine-needle aspiration (FNA) biopsy, revealing cryptococcosis and aspergillosis ( Fig. 3). The patient then asked to leave the hospital and was placed on oral voriconazole.

EUS-guided FNA has emerged as an important diagnostic imaging modality. It is

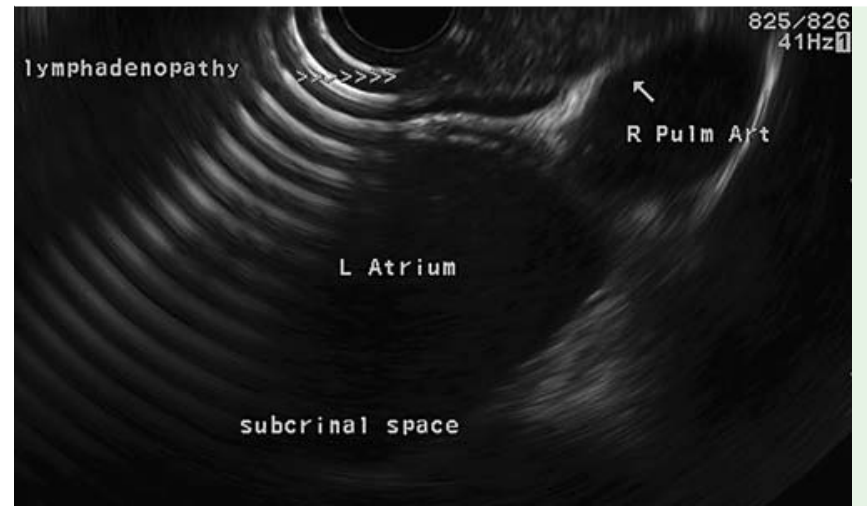

Fig. 2 Endoscopic ultrasound (EUS) revealing lymphadenopathy (arrows).

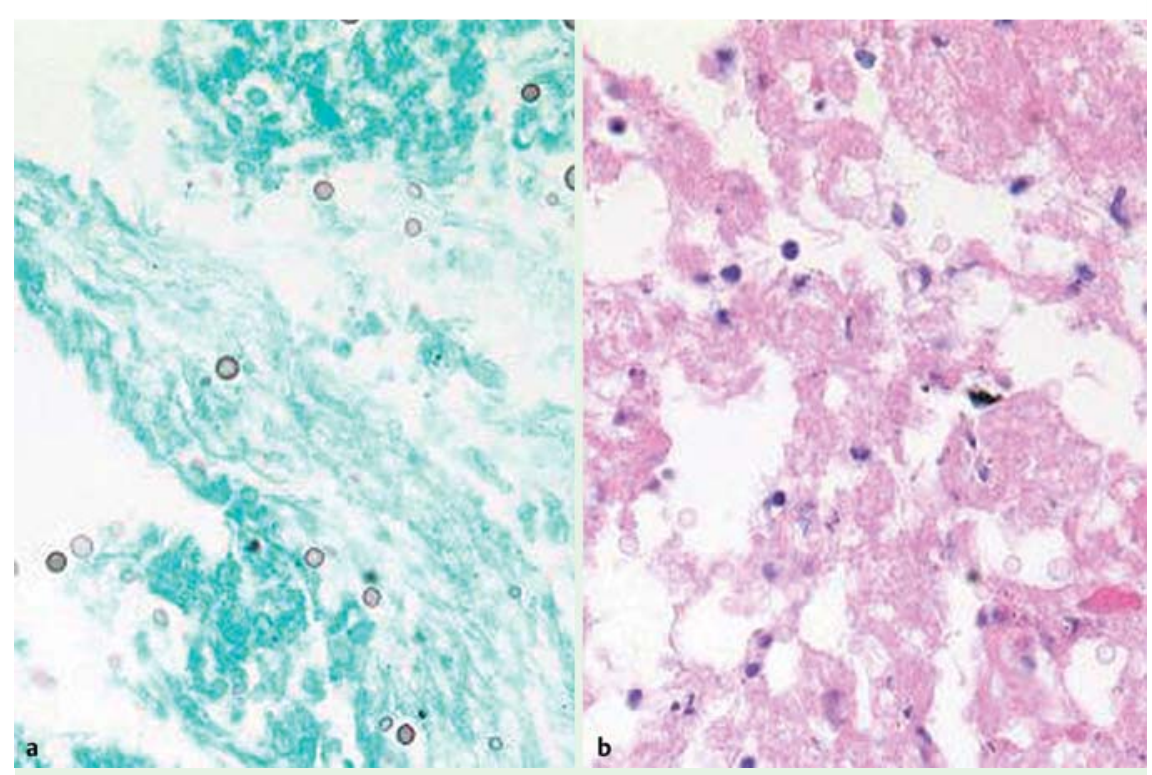

Fig. 3 Cryptococcus on histopathological staining using: a Grocott's methenamine silver $(40 \times)$; b hematoxylin and eosin (40×). (Aspergillus not shown.)

a non-surgical method to obtain tissue sampling from mediastinal lesions, with the advantage of real-time ultrasound guidance [1]. EUS can identify lymph nodes as small as $3 \mathrm{~mm}$, particularly in the celiac, subcarinal, and aorto-pulmonary areas [2]. Pulmonary cryptococcus has rarely been diagnosed via EUS-guided FNA. Concurrent diagnoses of patients with pulmonary cryptococcal isolates are common, especially in patients with HIV infection [3]. Here, aspergillus was concomitantly found. It has been shown that EUS with FNA has a $97 \%$ accuracy rate for tissue confirmation, and should be the test of choice especially for evaluating posterior mediastinal lymphadenopathy $[4,5]$.
In conclusion, we report a non-traditional modality to diagnose pulmonary cryptococcus using EUS with FNA.

\section{Endoscopy_UCTN_Code_CCL_1AF_2AC}

\section{Competing interests: None}

\section{E. Rahimi ${ }^{1}$, D. Behin ${ }^{2}$, A. Pellecchia ${ }^{3}$}

Department of Internal Medicine, Jacobi Medical Center, Bronx, New York, USA

2 Department of Gastroenterology, Montefiore Medical Center, Bronx, New York, USA

Department of Gastroenterology, Jacobi Medical Center, Bronx, New York, USA 


\section{References}

1 Puri R, Vilmann P, Sud R, Kumar M et al. Endoscopic ultrasound-guided fine-needle aspiration cytology in the evaluation of suspected tuberculosis in patients with isolated mediastinal lymphadenopathy. Endoscopy 2010; 42: 462-467

2 Prasad P, Wittmann J, Pereira S. Endoscopic ultrasound of the upper gastrointestinal tract and mediastinum: diagnosis and therapy. Cardiovasc Intervent Radiol 2006; 29 : 947-957

3 Lanjewar DN, Duggal R. Pulmonary pathology in patients with AIDS: an autopsy study from Mumbai. HIV Med 2001; 2: 266-271
4 Puli SR, Batapati Krishna Reddy J, Bechtold $M L$ et al. Endoscopic ultrasound: its accuracy in evaluating mediastinal lymphadenopathy? A meta-analysis and systematic review. World J Gastroenterol 2008; 14: $3028-3037$

5 Eloubeidi MA, Cerfolio RJ, Chen VK et al. Endoscopic ultrasound-guided fine needle aspiration of mediastinal lymph node in patients with suspected lung cancer after positron emission tomography and computed tomography scans. Ann Thorac Surg 2005; 79: $263-268$
Bibliography

DOI $10.1055 / \mathrm{s}-0030-1256616$

Endoscopy 2011; 43: E304 -E305

(c) Georg Thieme Verlag KG Stuttgart · New York . ISSN 0013-726X

\section{Corresponding author}

E. Rahimi, MD

Jacobi Medical Center/Albert Einstein College of Medicine

Department of Internal Medicine 1400 Pelham Parkway

Bronx

New York 10461

USA

Fax: +1-718-918-7640

erikrahimi@yahoo.com 